\title{
Predictive Base Station Activation in Futuristic Energy-Efficient Control/Data Separated RAN
}

\author{
Abdelrahim Mohamed*, Oluwakayode Onireti ${ }^{\dagger}$, Muhammad $\operatorname{Imran}^{\dagger}$, Haris Pervaiz ${ }^{*}$, Pei Xiao*, Rahim Tafazolli* \\ *Institute for Communications Systems (ICS), Home of 5GIC, University of Surrey, Guildford, UK \\ ${ }^{\dagger}$ School of Engineering, University of Glasgow, Glasgow, UK \\ E-mail: abdelrahim.mohamed @ surrey.ac.uk
}

\begin{abstract}
Nowadays, system architecture of the fifth generation (5G) cellular system is becoming of increasing interest. To reach the ambitious 5G targets, a dense base station (BS) deployment paradigm is being considered. In this case, the conventional always-on service approach may not be suitable due to the linear energy/density relationship when the BSs are always kept on. This suggests a dynamic on/off BS operation to reduce the energy consumption. However, this approach may create coverage holes and the BS activation delay in terms of hardware transition latency and software reloading could result in service disruption. To tackle these issues, we propose a predictive BS activation scheme under the control/data separation architecture (CDSA). The proposed scheme exploits user context information, network parameters, BS sleep depth and measurement databases to send timely predictive activation requests in advance before the connection is switched to the sleeping BS. An analytical model is developed and closed-form expressions are provided for the predictive activation criteria. Analytical and simulation results show that the proposed scheme achieves a high BS activation accuracy with low errors w.r.t. the optimum activation time.
\end{abstract}

Index Terms-5G cellular system; context awareness; control/data separation architecture; energy efficiency; predictive activation; radio access network.

\section{INTRODUCTION}

In the foreseeable future, network densification will become one of the dominant themes to support enormous capacity and massive connectivity [1]. Such deployment scenarios raise several challenges and they impose new constraints. Among others, energy efficiency will become a critical consideration. A breakdown of the cellular network suggests that it is of vital importance to reduce the base station (BS) energy consumption, since more than $80 \%$ of the access network power is consumed by the BSs [2]. The conventional alwayson service concept may not be suitable for the future radio access network (RAN), since energy consumption of the RAN increases linearly with the number of active BSs [3]-[5]. This suggests a paradigm shift towards on demand systems with BS sleep modes, which can significantly improve the (almost) load-independent power consumption profile that characterises small, e.g., pico, BSs of current cellular networks.

In this context, several research papers have investigated the BS sleep modes, and a key conclusion is drawn on the importance of network-driven sleep mode mechanisms. In this case, a macro BS assists the on/off decisions of the small BSs within its coverage in a two-tier RAN architecture. This approach has been found as the most promising sleep mode scenario that guarantees ubiquitous connectivity whilst providing the highest energy saving gains [4], [5]. Despite the potential benefits, all sleep mode mechanisms are restricted by two main challenges: 1) All BSs, irrespective of their activity state, have to transmit periodic pilot signals for measurement purposes, which limits the achievable energy saving gains. 2) The transition delay between sleep and active states may result in service disruption which in turns may prevent the network from delivering the required quality of service (QoS).

The first problem, i.e., the periodic transmission of pilot signals for measurement purposes, limits the sleep duration of the BS. These measurements are required by idle user equipment (UE) for cell (re)selection, and by active UE for handover decisions. Suspending the periodic transmission of pilot signals in the sleeping BSs increases the sleep period and the energy saving gains. However, the cell (re)selection and handover processes will exclude these BSs from the candidate set, resulting in suboptimal serving node selection decisions. On the other hand, the transition delay between sleep and active states depends on the BS sleep depth. A BS in a deep sleep level (i.e., most of the BS components are switched off) consumes less energy and requires more time to be activated as compared with a BS in a light sleep level. In other words, an energy/delay trade-off is expected when sleep modes are employed. Based on the current technology and hardware design, this delay is non-negligible and it has a direct impact on the feasible time scale for the BS on/off adaptation.

In this paper, we tackle these two challenging issues by proposing a predictive measurement and activation scheme for BSs in the sleep mode. A futuristic RAN architecture with separation of control and data planes is considered due to its unique features and intrinsic energy-efficient design. The proposed approach predicts the sleeping BS to be activated in advance, thus allowing timely on/off decisions whilst minimising the activation duration. The predictions are made based on the measurement databases for sleeping BSs, UE measurement for active BSs and information reported from the BSs. The reminder of this paper is structured as follows: Section II presents the considered network architecture along with a high level overview of the proposed approach. Section III develops the sleeping BS activation prediction model and formulates the data plane BS activation criteria. Section IV provides and discusses numerical and simulation results that assess the performance of the proposed scheme. Finally, Section V concludes the paper.

\section{Architecture AND Proposed ApproACH}

\section{A. Control/Data Separation Architecture}

The control/data separation architecture (CDSA) is considered in this paper. It depends on separating the signals required for full coverage from those needed to support high data rate transmission, resulting in a two tier RAN architecture with a logical separation between control and data planes. In the CDSA, an idle UE maintains a single connection with a 


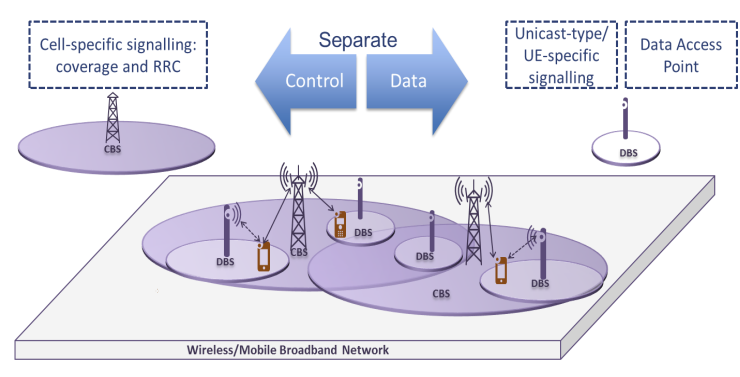

Figure 1: Control/Data Separation Architecture (CDSA)

control base station (CBS) for network connectivity as shown in Fig. 1. When the UE switches to the active mode, e.g., starting a session or receiving a call, it establishes a high rate connection with a data base station (DBS) whilst maintaining the low rate connection with the CBS for signalling, radio resource control (RRC) and mobility management.

A mapping that illustrates the functionalities supported by each plane can be found in [6, Table II] and [7, Table I]. The CBSs guarantee a low rate coverage layer that provides necessary signalling related to cell search and acquisition of system information. Broadcast/multicast services, paging functionalities and serving DBS selection are also provided by the CBS. This allows switching off the DBSs as long as they are not needed for data transmission. In addition, the CDSA enables exploiting the large footprint of the CBS and its wider view of network status and parameters which could result in optimised resource selection. During the active session, the DBS provides data transmission along with the necessary signalling for channel estimation, link adaptation and beamforming. At the same time, the CBS serves as an anchor point and handles signalling, mobility management and DBS on/off decisions [8].

\section{B. Proposed Approach: High Level Overview}

We focus on minimising energy consumption of the DBSs only, while the CBSs are always kept on. The rationale behind this scheme originates from the following facts:

- The CBSs provide network connectivity. Consequently, the requirement to maintain an anywhere/anytime service concept prevents switching off the CBSs.

- Energy consumption of the CBSs (which are typically macro cells) scales to some extent with the traffic load, unlike the nearly load-independent energy consumption profile of the DBSs (which are typically small cells) [9].

- The DBSs provide data transmission to active UE only. This transmission offers several adaptation and energy saving opportunities in the DBS layer.

- The DBS density is significantly higher than the CBS density. Thus energy consumption of the DBS layer becomes the main source of inefficiency in dense deployment scenarios.

When a DBS is in the sleep mode, the transmission circuitry is switched off and the pilot signal is suspended to increase the sleep period and the energy saving gains. Suspending the pilot transmission makes the DBS invisible to the UE, resulting in suboptimal serving node selection decisions (when there are other active DBSs that can serve the UE) or it may result in disconnecting the data plane link (when the sleeping DBS is the only candidate to serve the UE). The

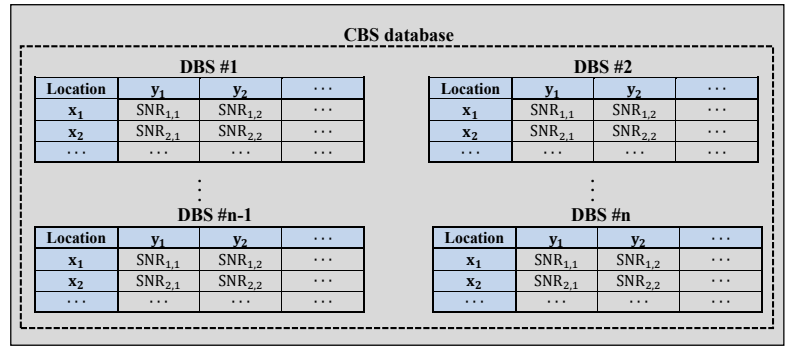

Figure 2: Pilot measurement database located at the CBS [6]

CDSA offers a solution to this problem by using measurement databases for sleeping DBSs. This requires each CBS to be equipped with a database that contains measurement reports of each DBS within its footprint. These databases can hold signal strength measures such as reference signal received power (RSRP) and signal-to-noise ratio (SNR), or it can hold signal quality measures such as reference signal received quality (RSRQ) and signal-to-interference-plus-noise ratio (SINR).

In this paper, we consider the signal strength measures due to their low storage, processing and updating requirements. A conceptual example of this database is shown in Fig. 2. Based on current standards such as the 3GPP measurement reporting and control mechanism [10], each UE reports measurements of the serving DBS and the detectable DBSs to the CBS. In addition, the UE position is reported along with the measurements. The CBS stores these measurements in the database and maps them to the location where they are reported from. The procedure for updating the database entries when there are previous measurements from the location being reported by the UE can be found in [11] and [12]. Based on the database approach, all the DBSs become visible to the UE even if they are switched off and do not transmit pilot signals. This can be realised by measuring the active DBSs signals and using the stored measurements for the sleeping DBSs. Such an approach can be viewed as a collaborative serving node selection scheme where each UE utilises measurements of other UE whilst maintaining the DBSs in a sleep mode (as long as they are not needed for data transmission).

Consider a DBS at a certain sleep level which requires $T_{\text {on }}$ time to be activated (i.e., the DBS activation duration). The latter also includes the time to determine the best serving small cell (typically $10 \mathrm{~ms}$ in the database approach). If the target DBS to be activated and the exact time when the connection will be switched to the target DBS are known, then the CBS can send an activation request to the DBS at least $T_{\text {on }}$ time in advance before the connection is switched. Such an advanced DBS activation request avoids service disruption and guarantees the QoS. Nonetheless, the DBS to be activated and the exact time when the connection will be switched to the target DBS are usually unknown until the switching criteria is satisfied. In this case, the DBS activation duration may cause service disruption and the UE may loose the data plane connection until the DBS is fully activated.

The proposed scheme predicts the DBS to be activated and sends the activation request in advance, thus enabling timely DBS activation decisions whilst guaranteeing the QoS. The basic idea depends on exploiting the DBS pilot measurements (measured for the active DBSs, and obtained from the database for sleeping DBSs) to predict future measurements. 
Such a prediction enables the CBS to send advance activation requests to the sleeping DBS. We assume that the sleep level (i.e., the sleeping depth and the activation duration $T_{o n}$ ) of each DBS is known by the underlay CBS. This information can be periodically exchanged through the CBS/DBS backhaul links. The predictive activation scheme exploits measurement trends coupled with UE contextual information (i.e., location, direction and speed) to predict the target DBS to be activated and the appropriate time to send the activation request. Such a prediction enables activating the sleeping DBS (if necessary) before the UE visits it, thus avoiding the activation delay when the data plane connection is switched from the previous (i.e., the serving) DBS to the new (i.e., the target) DBS.

\section{Activation Prediction Scheme}

The activation prediction scheme depends on a measurement prediction performed by either the UE or the CBS. The UE periodically measures signals of the active DBSs at every measurement gap as defined in current standards such as the long term evolution (LTE) [13]. On the other hand, the sleeping DBS measurements are obtained from the database located at the CBS which takes the UE location coordinates as an input. A trending mechanism is proposed to predict future measurements based on the rate of measurement change. The predicted measurements are used to predict the DBS to be activated as well as the appropriate time to send the activation request.

We consider DBSs with a conventional hysteresis-based serving node selection mechanism, where the UE-DBS connection is switched from the serving DBS to the target DBS if the following condition is true:

$$
\log _{10}\left(P_{2, t}\right) \geq \log _{10}\left(P_{2, s}\right)+\log _{10}(H),
$$

where $P_{2, t}$ is the received power from the target DBS ' $t$ ', $P_{2, s}$ is the received power from the serving DBS ' $s$ ', and $H$ is the handover hysteresis. In the rest of this paper, the subscripts $s$, $t$ and $u$ are used to denote parameters of the serving DBS, the target DBS and the UE, respectively. It is worth emphasising that the proposed scheme can be applied to other serving node selection strategies with simple modifications, and the criteria of (1) is considered here as an example. Initially, we assume that the target DBS and the time when the condition of (1) will be satisfied are known by the CBS. Consequently, in Section III-A we derive the serving DBS signal level at which the CBS sends the activation request to the target DBS before the condition of (1) is satisfied. In Section III-B, the assumptions on the CBS knowledge are relaxed and a model is developed to predict the target DBS to be activated and the time when the condition of (1) will be satisfied.

\section{A. Activation Request Triggering Model}

The CBS decision to send the activation request to the DBS is non-trivial even if the target DBS for the activation request and the exact time when the condition of (1) will be satisfied are known in advance by the CBS. An early activation request may guarantee the QoS but it increases the energy consumption since the target DBS is switched on and becomes active without being used until the UE visits this DBS. On the other hand, a late activation request reduces the energy consumption, however this could come at the expense of degrading the QoS because the target DBS may not become

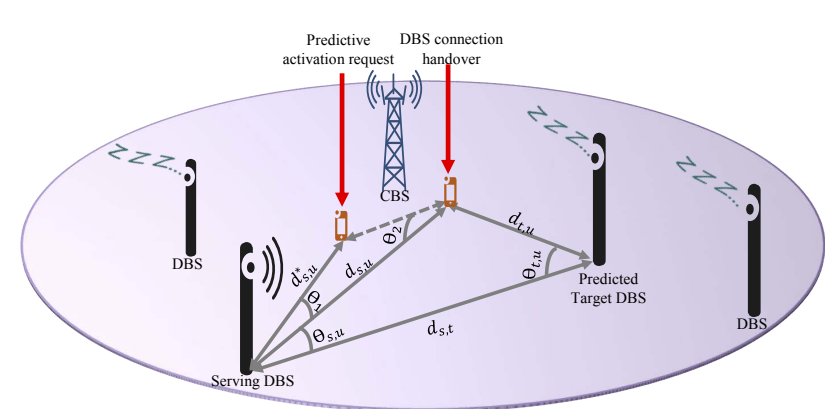

Figure 3: Distance and angle parameters of the predictive activation request scheme

fully active before the UE visits this DBS. In this direction, we derive the optimum serving DBS signal level at which the CBS sends the activation request to the target DBS.

Considering the data plane connection switching criteria defined in (1), and a generic path loss model of $c d^{\alpha}$ with similar shadowing characteristics for the paths for simplicity, where $c$ is the distance-independent path loss component, $d$ is the distance, and $\alpha$ is the path loss exponent. Then, the condition of (1) can be expressed as:

$$
\log _{10}\left(\frac{P_{1, t}}{c d_{t, u}^{\alpha}}\right) \geq \log _{10}\left(\frac{P_{1, s}}{c d_{s, u}^{\alpha}}\right)+\log _{10}(H),
$$

where $P_{1, t}$ is the transmit power of the target DBS, $P_{1, s}$ is the transmit power of the serving DBS, $d_{t, u}$ is the distance between the target DBS and the UE, $d_{s, u}$ is the distance between the serving DBS and the UE, and the transmitter/receiver antenna gain is normalised to unity. In a linear form, (2) can be rewritten as:

$$
\frac{P_{1, t}}{c d_{t, u}^{\alpha}} \geq \frac{P_{1, s} H}{c d_{s, u}^{\alpha}} .
$$

The distance between the serving DBS and the target DBS denoted by $d_{s, t}$ can be expressed as:

$$
d_{s, t}=d_{s, u} \cos \left(\theta_{s, u}\right)+d_{t, u} \cos \left(\theta_{t, u}\right),
$$

where $\theta_{s, u}$ is the angle between the line connecting the UE with the serving DBS and the line connecting the serving DBS with the target DBS, $\theta_{t, u}$ is the angle between the line connecting the UE with the target DBS and the line connecting the serving DBS with the target DBS. These parameters are shown conceptually in Fig. 3 . The distance $d_{t, u}$ can be obtained by rearranging (4), i.e.,

$$
d_{t, u}=\frac{d_{s, t}-d_{s, u} \cos \left(\theta_{s, u}\right)}{\cos \left(\theta_{t, u}\right)} .
$$

Consider the case that the CBS decided to send the activation request when the serving DBS signal reaches $P_{2, s}^{*}$ and the UE is located at a distance of $d_{s, u}^{*}$ from the serving DBS. Thus the distance between the UE and the serving DBS when the condition of (1) is satisfied can be written as:

$$
d_{s, u}=d_{s, u}^{*} \cos \left(\theta_{1}\right)+v_{u} T_{r} \cos \left(\theta_{2}\right)
$$

where $v_{u}$ is the UE velocity, $T_{r}$ is the time duration between the instance when the activation request is sent and the instance when the condition of (1) is satisfied, $\theta_{1}$ is the angle between the line connecting the serving DBS with the UE when the activation request is sent and the line connecting the 
serving DBS with the point where the handover is predicted to happen as shown in Fig. 3, $\theta_{2}$ is the angle between the line connecting the UE when the activation request is sent with the point where the handover is predicted to happen and the line connecting the serving DBS with the point where the handover is predicted to happen, assuming that the UE does not change its direction and speed after the activation request is sent. Substituting (6) into (5) yields:

$$
d_{t, u}=\frac{d_{s, t}-\left(d_{s, u}^{*} \cos \left(\theta_{1}\right)+v_{u} T_{r} \cos \left(\theta_{2}\right)\right) \cos \left(\theta_{s, u}\right)}{\cos \left(\theta_{t, u}\right)} .
$$

Subsequently, the condition of (3) can be rewritten as is in (8) on top of the next page, by substituting (7) and (6) in (3). Solving (8) for $T_{r}$ yields (9) on top of the next page.

For a target DBS at a certain sleep level with activation duration $T_{o n}$, the CBS should send the activation request at least $T_{\text {on }}$ time in advance before the condition of (1) is satisfied. The condition for sending the activation request can be expressed as:

$$
T_{r} \geq T_{\text {on }}
$$

Substituting (9) into the left hand side of (10) and solving the resultant equation for $d_{s, u}^{*}$ as in (11) on top of next page yields the maximum UE distance from the serving DBS below which the activation request should be sent to the target DBS. Finally, $P_{2, s}^{*}$ can be formulated as the ratio between the transmit power of the serving DBS and the path loss at a distance of (11), the exact closed form expression is given by (12) on top of next page.

It can be noticed that the condition of (12) depends on network parameters that can be easily obtained by the CBS. For instance, the serving and the target DBSs transmit power, the handover and serving node selection hysteresis, the target DBS sleep level, and the distance between the serving and the target DBSs are network-specific rather than UE-specific parameters that can be gathered by the CBS through backhaul links. On the other hand, $c$ and $\alpha$ depend on the environment and they can be estimated when the DBS transmit power is known by the UE. In addition, the UE speed and direction w.r.t. the serving and the target DBSs can be periodically reported by the UE to the CBS through the low rate control plane link. Thus the CBS is able to evaluate the condition of (12) with the aid of the known network parameters and the UE context. Nonetheless, condition (12) assumes that the CBS knows the target DBS in advance. The following section relaxes this assumption and develops a model to predict the target DBS for the activation request.

\section{B. Prediction of the Target DBS for Activation}

The prediction of the target DBS to be activated depends on measurement trending and rate of change. The Grey system theory is adopted as the trending mechanism due to its ability to average fluctuations of the input data sequence. Although filters are used at the UE to minimise the effect of these fluctuations, the measured signals will still suffer from fluctuations after the conventional filtering process due to fast fading as well as the changing environment when surrounding users and objects are not static. Thus a trending mechanism, such as the Grey theory, with intrinsic averaging becomes important to improve the prediction outcome whilst removing the fluctuation effect. The basic concept depends on translating the data sequence into a monotonic increasing function, and representing this function by a differential equation and solving it to find the model's parameters. For the signal measurement prediction, a $\operatorname{GM}(1,1)^{1}$ Grey model [14] can be constructed for each DBS as:

- Measurement, i.e., received signal power, sequence of each DBS (measured by the UE for active DBSs, and obtained from the database for sleeping DBSs) is represented as a time series given by:

$$
P_{2}^{\langle 0\rangle}(i)=\left(P_{2}^{\langle 0\rangle}(1), P_{2}^{\langle 0\rangle}(2), \ldots, P_{2}^{\langle 0\rangle}(n)\right)
$$

where the superscript $\langle 0\rangle$ means original measurements (i.e., before processing), $i=1,2,3, \ldots, n$ is the measurement index and $n$ is the number of measurements (per DBS) used in the prediction process. Notice that this process is performed for the serving DBS and all neighbouring DBSs, thus the subscript of the signal measurement is unified in this section.

- An accumulated generating operation (AGO) translates $P_{2}^{\langle 0\rangle}(i)$ to a monotonic increasing function $P_{2}^{\langle 1\rangle}(i)$ as:

$$
\begin{aligned}
P_{2}^{\langle 1\rangle}(i) & =\operatorname{AGO}\left\{P_{2}^{\langle 0\rangle}(i)\right\} \\
& =\left(P_{2}^{\langle 0\rangle}(1), \sum_{i=1}^{2} P_{2}^{\langle 0\rangle}(i), \ldots, \sum_{i=1}^{n} P_{2}^{\langle 0\rangle}(i)\right) .
\end{aligned}
$$

- Based on (14), an inverse accumulated generating operation (IAGO) can be formulated as:

$$
P_{2}^{\langle 1\rangle}(i)=P_{2}^{\langle 1\rangle}(i-1)+P_{2}^{\langle 0\rangle}(i) .
$$

- The $\operatorname{GM}(1,1)$ model is defined by the following equation [14]:

$$
\frac{\mathrm{d} P_{2}^{\langle 1\rangle}}{\mathrm{d} u}+a P_{2}^{\langle 1\rangle}=b,
$$

where $a$ is the develop parameter and $b$ is the Grey input. The solution to (16) at time index $i$ is:

$$
\begin{aligned}
P_{2}^{\langle 1\rangle}(i+1) & =\left(P_{2}^{\langle 1\rangle}(1)-\frac{b}{a}\right) \mathrm{e}^{-a i}+\frac{b}{a} \\
& =\left(P_{2}^{\langle 0\rangle}(1)-\frac{b}{a}\right) \mathrm{e}^{-a i}+\frac{b}{a} .
\end{aligned}
$$

By substituting the IAGO of (15) into (17), the predicted signal one measurement gap in advance $\bar{P}_{2}^{\langle 0\rangle}(i+1)$ can be expressed as:

$$
\bar{P}_{2}^{\langle 0\rangle}(i+1)=\mathrm{e}^{-a i}\left(1-\mathrm{e}^{a}\right)\left(P_{2}^{\langle 0\rangle}(1)-\frac{b}{a}\right) .
$$

Similarly, the predicted signal at $j$ measurement gaps in advance can be formulated as:

$$
\bar{P}_{2}^{\langle 0\rangle}(i+j)=\mathrm{e}^{-a(i+j-1)}\left(1-\mathrm{e}^{a}\right)\left(P_{2}^{\langle 0\rangle}(1)-\frac{b}{a}\right) .
$$

Equation (19) can be used to predicted a series of signal measurements. However, the model parameters $a$ and $b$ need to be calculated before the prediction is performed. These

\footnotetext{
${ }^{1}$ the first 1 means the model uses first order differential equations, while the second 1 means there is one variable.
} 


$$
\begin{gathered}
P_{1, t}\left(\frac{\cos \left(\theta_{t, u}\right)}{d_{s, t}-\left(d_{s, u}^{*} \cos \left(\theta_{1}\right)+v_{u} T_{r} \cos \left(\theta_{2}\right)\right) \cos \left(\theta_{s, u}\right)}\right)^{\alpha} \geq \frac{P_{1, s} H}{\left(d_{s, u}^{*} \cos \left(\theta_{1}\right)+v_{u} T_{r} \cos \left(\theta_{2}\right)\right)^{\alpha}} \\
T_{r}=\frac{\left(\frac{P_{1, s} H}{P_{1, t}}\right)^{\frac{1}{\alpha}}\left(d_{s, t}-d_{s, u}^{*} \cos \left(\theta_{s, u}\right) \cos \left(\theta_{1}\right)\right)-d_{s, u}^{*} \cos \left(\theta_{t, u}\right) \cos \left(\theta_{1}\right)}{v_{u} \cos \left(\theta_{2}\right)\left(\cos \left(\theta_{t, u}\right)+\left(\frac{P_{1, s} H}{P_{1, t}}\right)^{\frac{1}{\alpha}} \cos \left(\theta_{s, u}\right)\right)} . \\
d_{s, u}^{*} \leq \frac{d_{s, t}}{\cos \left(\theta_{1}\right)\left(\cos \left(\theta_{s, u}\right)+\left(\frac{P_{1, t}}{P_{1, s} H}\right)^{\frac{1}{\alpha}} \cos \left(\theta_{t, u}\right)\right)}-\frac{T_{o n} v_{u} \cos \left(\theta_{2}\right)}{\cos \left(\theta_{1}\right)} . \\
P_{2, s}^{*} \geq \frac{P_{1, s}}{c}\left[\frac{\cos \left(\theta_{1}\right)\left(\cos \left(\theta_{s, u}\right)+\left(\frac{P_{1, t}}{P_{1, s} H}\right)^{\frac{1}{\alpha}} \cos \left(\theta_{t, u}\right)\right)}{d_{s, t}-T_{o n} v_{u} \cos \left(\theta_{2}\right)\left(\cos \left(\theta_{s, u}\right)+\left(\frac{P_{1, t}}{P_{1, s} H}\right)^{\frac{1}{\alpha}} \cos \left(\theta_{t, u}\right)\right)}\right]^{\alpha} .
\end{gathered}
$$

parameters can be obtained by expressing the derivative in (16) as:

$$
\frac{\mathrm{d} P_{2}^{\langle 1\rangle}}{\mathrm{d} u} \rightarrow P_{2}^{\langle 1\rangle}(i+1)-P_{2}^{\langle 1\rangle}(i),
$$

and the right hand side of (20) can be replaced with $P_{2}^{\langle 0\rangle}(i+1)$ based on the IAGO of (15), i.e.,

$$
\frac{\mathrm{d} P_{2}^{\langle 1\rangle}}{\mathrm{d} u} \rightarrow P_{2}^{\langle 0\rangle}(i+1) .
$$

The mean value of adjacent measurements is:

$$
z^{\langle 1\rangle}(i)=\frac{1}{2} P_{2}^{\langle 1\rangle}(i)+\frac{1}{2} P_{2}^{\langle 1\rangle}(i-1) \rightarrow P_{2}^{\langle 1\rangle}(u) .
$$

Based on (21) and (22), the Grey differential equation of (16) can be rewritten as:

$$
P_{2}^{\langle 0\rangle}(i)+a z^{\langle 1\rangle}(i)=b .
$$

Rearranging (23) and writing the resultant equation in a matrix form yields:

$$
\left[\begin{array}{c}
P_{2}^{\langle 0\rangle}(2) \\
P_{2}^{\langle 0\rangle}(3) \\
\vdots \\
P_{2}^{\langle 0\rangle}(n)
\end{array}\right]=\left[\begin{array}{cc}
-z^{\langle 1\rangle}(2) & 1 \\
-z^{\langle 1\rangle}(3) & 1 \\
\vdots & \vdots \\
-z^{\langle 1\rangle}(n) & 1
\end{array}\right] \cdot\left[\begin{array}{l}
a \\
b
\end{array}\right]
$$

finally, $a$ and $b$ can be obtained by solving (24), i.e.,

$$
\left[\begin{array}{c}
a \\
b
\end{array}\right]=\left[\begin{array}{cc}
-z^{\langle 1\rangle}(2) & 1 \\
-z^{\langle 1\rangle}(3) & 1 \\
\vdots & \vdots \\
-z^{\langle 1\rangle}(n) & 1
\end{array}\right]^{-1} \cdot\left[\begin{array}{c}
P_{2}^{\langle 0\rangle}(2) \\
P_{2}^{\langle 0\rangle}(3) \\
\vdots \\
P_{2}^{\langle 0\rangle}(n)
\end{array}\right]
$$

\section{Target DBS Selection}

When the serving DBS signal level reaches the lower bound of $P_{2, s}^{*}$ defined in (12), the CBS constructs the Grey model for the serving DBS and the neighbouring DBSs. Notice that $P_{2, s}^{*}$ will have several values because the UE direction w.r.t. to a certain neighbouring DBS will be different than the direction w.r.t. to the other neighbouring DBSs. In addition, the neighbouring DBSs may have different characteristics and they could be at different sleep levels. This results in a multiboundary DBS cell structure where each boundary represents a $P_{2, s}^{*}$ level. At each level, a target DBS is predicted. When the predicted DBS is not the same DBS that defines the $P_{2, s}^{*}$ level, the CBS waits until the next $P_{2, s}^{*}$ level is reached and repeats the process (i.e., in this case, the boundary is for a neighbouring DBS at a deeper sleep level, while the UE is moving towards a DBS at a lighter sleep level hence the activation request is delayed to maximise the DBS sleep period). Algorithm 1 illustrates the procedure followed by the CBS to select the target DBS for the activation request.

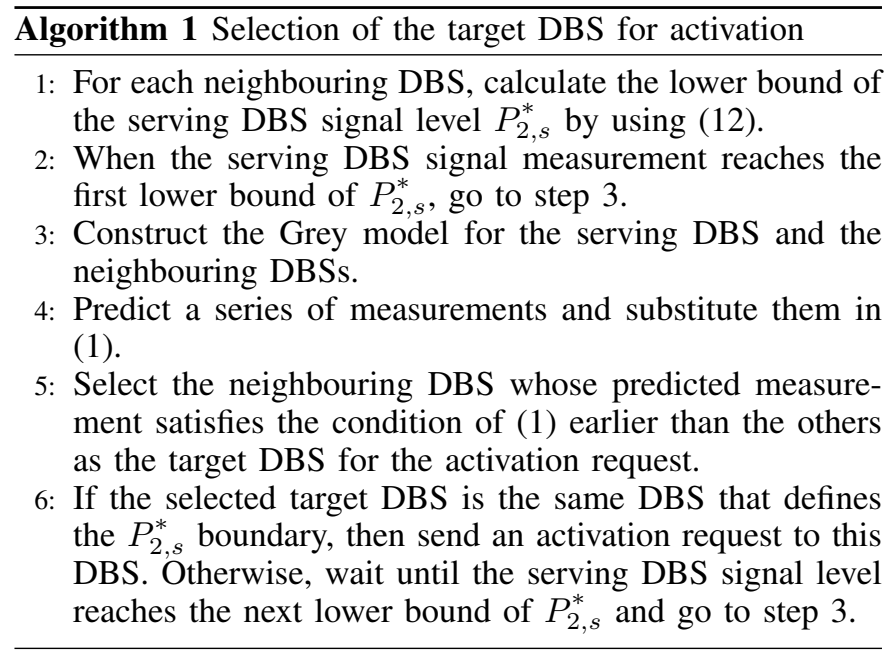

\section{Results and PeRformance Evaluation}

This section provides and discusses analytical and simulation results based on the proposed predictive activation request model. The $3 \mathrm{GPP}$ urban path loss model [15] has been considered and fitted to the generic model of Section III by using the least squares fitting method. With DBS height of $15 \mathrm{~m}$ and frequency of $2 \mathrm{GHz}$, the best-fit parameters are: $c=34.2925$ and $\alpha=3.76$. Fig. 4 compares the actual 3GPP 


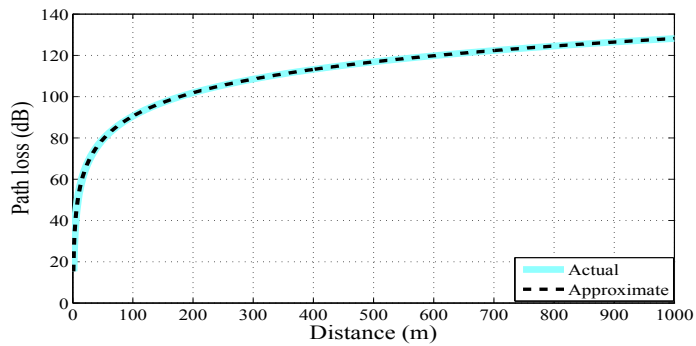

Figure 4: Actual 3GPP urban path loss model vs the fitted model

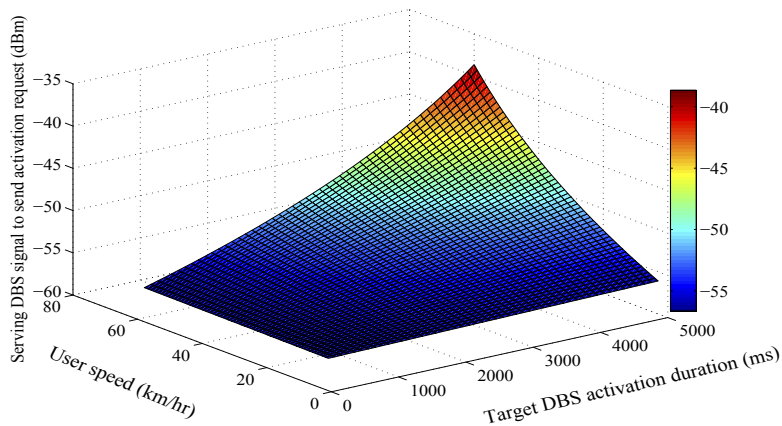

Figure 5: Effect of user speed and DBS sleep depth on the predictive activation request boundary

model and the approximate (i.e., fitted) model. As can be seen, the fitted model matches the actual model in the considered distance range. However, it is worth emphasising that the bestfit $c$ and $\alpha$ values depend on the distance range, i.e., a higher distance range requires recalculating the best-fit parameters.

Fig. 5 shows the effect of the UE speed and the target DBS sleep level, i.e., activation duration, on the lower bound of the serving DBS measurement $P_{2, s}^{*}$ to send the predictive activation request. The considered parameters are: serving and target DBS transmit power is $38 \mathrm{dBm}$, inter-site distance is $130 \mathrm{~m}$, hysteresis is $4 \mathrm{~dB}, \theta_{s, u}=20^{\circ}, \theta_{t, u}=50^{\circ}, \theta_{1}=\theta_{2}=0^{\circ}$. It can be noticed that as the UE speed increases, the CBS sends the predictive activation request to the target DBS at a higher serving DBS measurement level. Expressed differently, the predictive activation request is sent earlier for high speed users and delayed for low speed users. For instance, a UE with 50 $\mathrm{km} / \mathrm{hr}$ speed requires the CBS to send the predictive activation request when the serving DBS signal reaches $-43.4 \mathrm{dBm}$, for a target DBS at a sleep depth (i.e., activation duration) of 5000 $\mathrm{ms}$. On the other hand, the CBS can wait until the serving DBS signal reaches $-56 \mathrm{dBm}$ before sending the predictive activation request for a UE with $3 \mathrm{~km} / \mathrm{hr}$ speed. Although the CBS can send the activation request earlier for low speed users, delaying the activation request until the UE-specific lower measurement bound is reached maximises the target DBS sleep period.

In addition, Fig. 5 indicates that the target DBS sleep depth, i.e., the activation duration, has a significant impact on the CBS decision to send the activation request. As the DBS sleep depth increases, the CBS sends the activation request at a higher serving DBS measurement level. This ensures that the target DBS becomes fully active before the UE visits this DBS, thus enabling seamless and fast DBS-UE link switching.

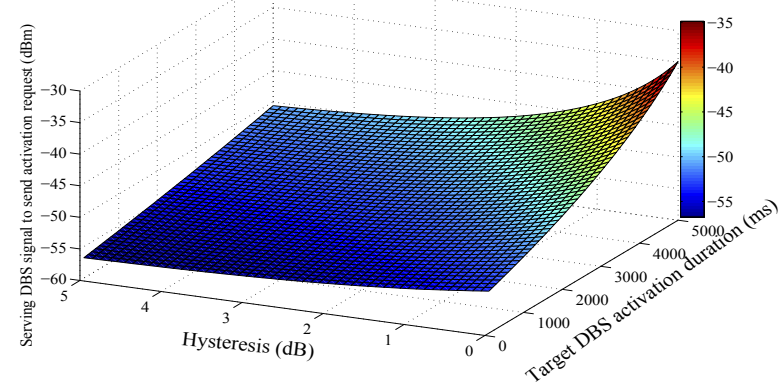

Figure 6: Effect of serving node selection hysteresis and DBS sleep depth on the predictive activation request boundary, for $30 \mathrm{~km} / \mathrm{hr} \mathrm{UE}$

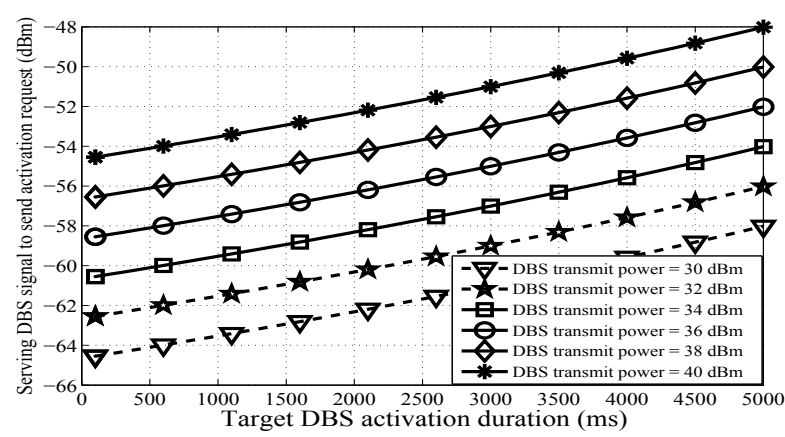

Figure 7: Effect of DBS transmit power and sleep depth on the predictive activation request boundary

An interesting finding that can be observed in Fig. 5 is that the CBS decision to send the predictive activation request becomes almost insensitive to the UE speed when the target DBS is in a very light sleep level, e.g., activation duration of $100 \mathrm{~ms}$. In this case, the activation request is sent to the target DBS at roughly the same boundary irrespective of the UE speed.

Fig. 6 shows the effect of the serving node selection hysteresis on the CBS decision to send the predictive activation request. It can be seen that the CBS delays the predictive activation request as the hysteresis increases. This can be traced to the fact that hysteresis delays the data plane switching process, i.e., the UE remains connected with the old DBS for a longer time. In other words, a higher hysteresis motivates keeping the target DBS in sleep mode for a longer time. It is worth mentioning that numerical results in Fig. 6 consider a constant UE angle w.r.t. the target DBS for all hysteresis values, hence the serving DBS measurement boundary below which the CBS sends the predictive activation request remains roughly constant when $H \geq 4 \mathrm{~dB}$.

Fig. 7 shows the effect of the serving and the target DBS transmit power and the sleep depth on the CBS decision to send the predictive activation request. As the DBS transmit power increases, the CBS sends the activation request at a higher measurement level. This can be linked to the equal transmit power setting considered for all DBSs, which results in increasing the DBS overlap area as the DBS transmit power increases. Although the CBS decision to send the activation request depends on the DBS transmit power, this does not affect the target DBS sleep period because the equal power setting means that the activation request will be sent from the same UE location (which will have a higher measurement 


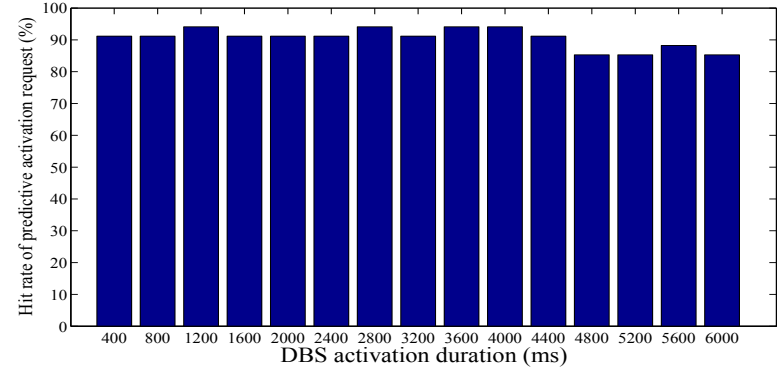

Figure 8: Hit rate of the predictive activation request

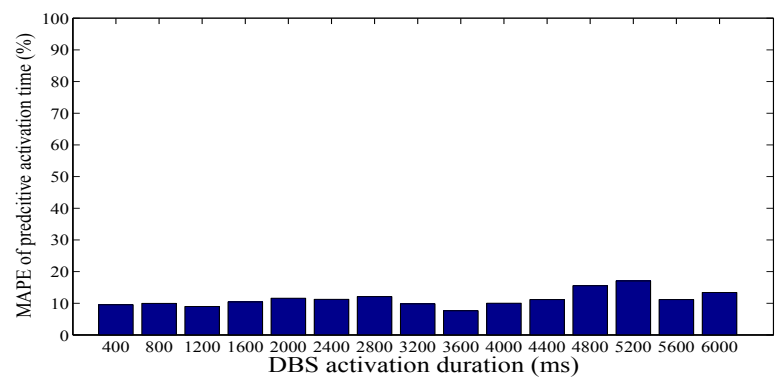

Figure 9: Mean absolute percentage error (MAPE) of the predictive activation time

level as the DBS transmit power increases). Thus it can be concluded that the UE speed and the DBS sleep depth are the most important parameters that have a significant impact on the CBS decision to activate the target DBS.

System level simulations have been performed to assess performance of the proposed scheme. The considered network consists of 19 DBSs under the control of a CBS. The 3GPP urban path loss model [15] has been considered along with the 3GPP typical urban channel model [16]. Each UE is moving with a constant speed of $10 \mathrm{~km} / \mathrm{hr}$. The DBS transmit power is $38 \mathrm{dBm}$ and inter-site distance is $130 \mathrm{~m}$. A measurement gap of $200 \mathrm{~ms}$ has been set. Fig. 8 shows hit rate of the predictive activation request (i.e., percentage of the correctly activated DBSs) for several DBS sleep depths while Fig. 9 provides the mean absolute percentage error (MAPE) of the predictive activation time. As can be observed, the proposed scheme achieves a hit rate of $85.3 \%-94.1 \%$ with a MAPE of $7.6 \%-17 \%$ w.r.t. the optimum activation time. The latter is defined as the time instance preceding the instance when the connection is switched towards the target DBS by $T_{o n}$ ms.

\section{CONCLUSION}

In this paper, a predictive BS activation scheme is proposed to minimise the effect of the BS activation delay when sleep modes are employed. The futuristic CDSA is considered, and the predictive activation scheme is proposed for the DBSs. The CDSA dual connection feature, the CBS wider view of network status and parameters, and the ability to maintain measurement databases at the CBS are exploited to avoid the periodic pilot transmission, thus overcoming one of the main limitations of conventional sleep mode mechanisms. Focusing on sleep modes in the DBS layer, a predictive activation scheme that takes into account network parameters, DBS sleep level and UE context information is proposed to predict the target DBS to be activated as well as the appropriate time to send the activation request. With the main objective of maximising the DBS sleep period, a lower bound for the DBS measurement level is derived, above which the activation request can be sent with relaxed constraints, and below which the UE may suffer QoS degradation if the target DBS is not reactivated. Analytical results indicate that the UE speed and the DBS sleep level have a significant impact on the activation boundary. System level simulation results show that the proposed scheme achieves a high hit rate in terms of correct activation requests with low errors w.r.t. the optimum activation time.

\section{ACKNOWLEDGEMENT}

This work is supported by Huawei under the project: Energy-Proportional eNodeB for LTE-Advanced and Beyond. We would like to acknowledge the support of the University of Surrey 5GIC members for this work.

\section{REFERENCES}

[1] J. G. Andrews et al., "What will 5G be?" IEEE Journal on Selected Areas in Communications, vol. 32, no. 6, pp. 1065-1082, June 2014.

[2] A. Fehske, G. Fettweis, J. Malmodin, and G. Biczok, "The global footprint of mobile communications: The ecological and economic perspective," IEEE Communications Magazine, vol. 49, no. 8, pp. 5562, August 2011

[3] E. Ternon, P. K. Agyapong, and A. Dekorsy, "Impact of varying traffic profile on phantom cell concept energy savings schemes," in 2015 IEEE 81st Vehicular Technology Conference (VTC Spring), May 2015, pp. 1 6.

[4] I. Ashraf, F. Boccardi, and L. Ho, "Sleep mode techniques for small cell deployments," IEEE Communications Magazine, vol. 49, no. 8, pp 72-79, August 2011 .

[5] _ - "Power savings in small cell deployments via sleep mode techniques," in Personal, Indoor and Mobile Radio Communications Workshops (PIMRC Workshops), 2010 IEEE 21st International Symposium on, Sept 2010, pp. 307-311.

[6] A. Mohamed, O. Onireti, M. Imran, A. Imran, and R. Tafazolli, "Control-data separation architecture for cellular radio access networks: A survey and outlook," IEEE Communications Surveys and Tutorials, vol. 18 , no. 1 , pp. 446-465, Firstquarter 2016.

[7] $\mathrm{X} . \mathrm{Xu}, \mathrm{G} . \mathrm{He}, \mathrm{S}$. Zhang, Y. Chen, and S. Xu, "On functionality separation for green mobile networks: Concept study over LTE," IEEE Communications Magazine, vol. 51, no. 5, pp. 82-90, May 2013.

[8] A. Mohamed, O. Onireti, M. Imran, A. Imran, and R. Tafazolli, "Predictive and core-network efficient RRC signalling for active state handover in RANs with control/data separation," IEEE Transactions on Wireless Communications, vol. 16, no. 3, pp. 1423-1436, March 2017.

[9] G. Auer et al., "How much energy is needed to run a wireless network?" IEEE Wireless Communications, vol. 18, no. 5, pp. 40-49, October 2011.

[10] 3GPP, "Evolved universal terrestrial radio access (E-UTRA); Radio resource control (RRC) protocol specification," Technical Specification, April 2016, 3GPP TS 36.331 version 13.1.0 Release 13. [Online]. Available: http://www.etsi.org/deliver/etsi_ts/136300_136399/ 136331/13.01.00_60/ts_136331v130100p.pdf

[11] E. Ternon, P. Agyapong, L. Hu, and A. Dekorsy, "Database-aided energy savings in next generation dual connectivity heterogeneous networks," in Proc. of IEEE Wireless Communications and Networking Conference, April 2014.

[12] A. Redondi, I. Filippini, and A. Capone, "Context management in energy-efficient radio access networks," in Proc. of 24th Tyrrhenian International Workshop on Digital Communications- Green ICT, September 2013, pp. 1-5

[13] 3GPP, "Evolved universal terrestrial radio access (E-UTRA); Requirements for support of radio resource management," Technical Sthe windows are the subject of a long term, transferrable guaranteepecification, March 2017, 3GPP TS 36.133 version 14.3.0.

[14] D. Julong, "Introduction to grey system theory," The Journal of grey system, vol. 1, no. 1, pp. 1-24, 1989.

[15] 3GPP, "Evolved Universal Terrestrial Radio Access (E-UTRA); Radio Frequency (RF) system scenarios," Technical Report, January 2016 3GPP TR 36.942 version 13.0.0 Release 13. [Online]. Available: http://www.3gpp.org/dynareport/36942.htm

[16] - "Technical Specification Group Radio Access Network; Deployment aspects," Technical Report, January 2016, 3GPP TR 25.943 version 13.0.0 Release 13. [Online]. Available: http://www.3gpp.org/DynaReport/25943.htm 